\title{
Role of PRY-1/Axin in heterochronic miRNA- mediated seam cell development
}

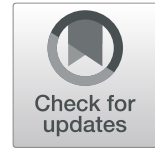

\author{
Avijit Mallick ${ }^{1}$, Ayush Ranawade ${ }^{1,2}$ and Bhagwati P. Gupta ${ }^{1 *}$ (D)
}

\begin{abstract}
Background: Caenorhabditis elegans seam cells serve as a good model to understand how genes and signaling pathways interact to control asymmetric cell fates. The stage-specific pattern of seam cell division is coordinated by a genetic network that includes WNT asymmetry pathway components WRM-1, LIT-1, and POP-1, as well as heterochronic microRNAs (miRNAs) and their downstream targets. Mutations in pry-1, a negative regulator of WNT signaling that belongs to the Axin family, were shown to cause seam cell defects; however, the mechanism of PRY1 action and its interactions with miRNAs remain unclear.

Results: We found that pry-1 mutants in C. elegans exhibit seam cell, cuticle, and alae defects. To examine this further, a miRNA transcriptome analysis was carried out, which showed that let-7 (miR-48, miR-84, miR-241) and lin-4 (lin-4, miR-237) family members were upregulated in the absence of pry-1 function. Similar phenotypes and patterns of miRNA overexpression were also observed in C. briggsae pry-1 mutants, a species that is closely related to $C$. elegans. RNA interference-mediated silencing of wrm-1 and lit-1 in the C. elegans pry-1 mutants rescued the seam cell defect, whereas pop-1 silencing enhanced the phenotype, suggesting that all three proteins are likely important for PRY-1 function in seam cells. We also found that these miRNAs were overexpressed in pop-1 hypomorphic animals, suggesting that PRY-1 may be required for POP-1-mediated miRNA suppression. Analysis of the let-7 and lin-4-family heterochronic targets, lin-28 and $h b l-1$, showed that both genes were significantly downregulated in pry1 mutants, and furthermore, lin-28 silencing reduced the number of seam cells in mutant animals.

Conclusions: Our results show that PRY-1 plays a conserved role to maintain normal expression of heterochronic miRNAs in nematodes. Furthermore, we demonstrated that PRY-1 acts upstream of the WNT asymmetry pathway components WRM-1, LIT-1, and POP-1, and miRNA target genes in seam cell development.
\end{abstract}

Keywords: C. elegans, C. briggsae, Pry-1, Axin, WNT asymmetry pathway, Seam cell, miRNA, Heterochronic development

\section{Background}

Caenorhabditis elegans hypodermal seam cells serve as a good model to elucidate spatiotemporal patterns of division and differentiation that enable cells to adopt sex-specific fates. They comprise two lateral rows of multipotent somatic cells which extend from the anterior to the posterior of the nematode (Fig. 1), and that divide in a stem cell-like manner to both self-renew, and generate daughter hypodermal, neuronal, and neuronal-support cells [1]. At the early L2 stage of development, the six seam cells (V1-6) undergo symmetric division to give rise to a total of 16 seam cells. By the end of the L4 stage, the cells terminally differentiate

\footnotetext{
* Correspondence: guptab@mcmaster.ca

'Department of Biology, McMaster University, ON L8S-4K1, Hamilton, Canada Full list of author information is available at the end of the article
}

and fuse with their neighbors to give rise to adult cuticular structures called alae [2].

Multiple genes and pathways have been shown to regulate seam cell division and differentiation. These include the lin-4 (lin-4 and miR-237) and let-7 (let-7, miR-48, miR84, and miR-241) families of heterochronic microRNAs (miRNAs) that regulate the relative timing of developmental events [3-7]. Specifically, lin-4 is required for the L1-L2 transition $[2,8]$, while the let-7 family members act at later stages of development [9]. Mutations in lin-4, miR-48, miR84 , and $m i R-241$ cause cells to reiterate stages, which ultimately affects both the number of differentiated seam cells, and cuticle development [9].

Previous studies have shown that heterochronic miRNAs regulate a number of targets, including hbl-1, lin-

(c) The Author(s). 2019 Open Access This article is distributed under the terms of the Creative Commons Attribution 4.0 International License (http://creativecommons.org/licenses/by/4.0/), which permits unrestricted use, distribution, and 


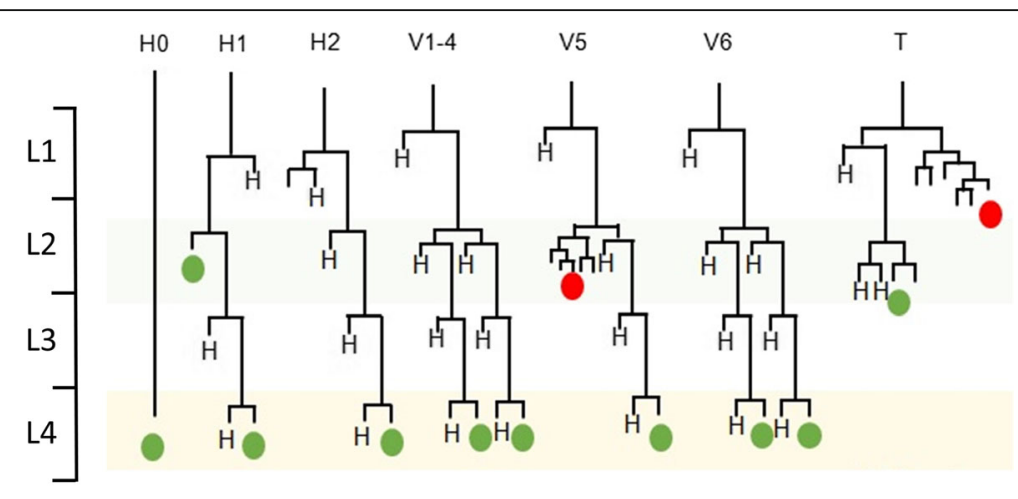

H- Hypodermal cell $\bigcirc$ - Seam cell $\bigcirc$ - Apoptotic cell death

Fig. 1 Seam cell asymmetric divisions during postembryonic development in C. elegans. During postembryonic development, C. elegans larvae undergo a series of molts, each of which is associated with carefully timed seam cell divisions. In the V1-4, and V6, cells undergo stem cell-like division where the anterior daughter fuses with the hyp7 syncytium. The posterior daughter then self-renews as another 'stem cell-like' seam cell. The exception to the asymmetric divisions is in early L2 stage where they undergo one symmetrical division prior to dividing again in an asymmetric manner

14, and lin-28 [10]. HBL-1 is a zinc-finger transcription factor that critically mediates embryogenesis [11], and that controls developmental timing during post-embryonic development [9]. LIN-14 is a novel class of transcription factor [12] that is initially expressed at high levels in hypodermal blast cells in newly hatched L1 animals but at lower levels by the L2 stage [13]. LIN-28 is a conserved RNA-binding protein that controls the maturation of let-7 miRNA [5, 14-16]. Hypomorphic and null alleles of lin-14 and $h b l-1$ cause an increase whereas lin-28 mutants cause a decrease in the overall number of seam cells $[9,17,18]$.

In addition to heterochronic miRNAs and their targets, seam cell division is also regulated by the divergent WNT asymmetry pathway, whose components include the $\beta$-catenins WRM- 1 and SYS-1, the NEMO-like kinase (NLK) LIT-1, and the T-cell factor/lymphoid enhancer factor (TCF/LEF) POP-1 [18, 19]. Removal of POP-1 activity causes seam cells to divide symmetrically, and thereby leads to an increase in their number. Conversely, since LIT-1 normally forms a complex with WRM-1 to phosphorylate and thus stimulate POP-1 export from the nucleus, disrupting WRM-1 and/or LIT-1 activity reduces the number of seam cells [19]. Similarly, the ratio of nuclear POP-1/SYS-1 activity affects the fate of daughter cells, such that those with lower POP-1 (and hence comparatively higher SYS-1) levels retain their seam cell fate, whereas those with higher POP-1 (and hence comparatively lower SYS-1) levels differentiate [20-23]. Genetic studies have also shown that WNT asymmetry pathway components interact with heterochronic genes to control seam cell development $[17,18]$.

While investigating the role of pry-1 in developmental and post-developmental processes, we observed that pry-1(mu38) animals exhibit weaker cuticle and abnormal alae. Further analysis revealed that they also display a higher number of seam cells, a phenotype that was previously reported [19]. Similar defects were also seen in a C. briggsae pry-1 mutant Cbr-pry-1(sy5353) [24], suggesting a conserved role for pry-1 in seam cell development and cuticular alae formation. These observations are in line with our recent pry-1(mu38) mRNA transcriptome profiling (RNA-Seq), which identified differentially expressed (DE) genes associated with 'cuticle development' [25]. Given that the heterochronic pathway involves both protein-coding and miRNA genes, in the present study we conducted an miRNA-specific whole genome RNA-Seq experiment, which uncovered six DE miRNAs that include members of lin-4 and let-7 families. To understand the interaction of $p r y-1$ with miRNAs during seam cell development, we knocked-down WNT asymmetry pathway components. Reducing wrm-1 and lit-1 expression suppressed, while silencing pop-1 exacerbated the pry-1 phenotype. Furthermore, an miRNA expression analysis conducted in a pop-1 hypomorph revealed a similar upregulation of miRNAs to that observed in pry-1(mu38) worms, suggesting both that POP-1 is critical for asymmetric seam cell division, and that its nuclear levels are likely reduced in pry-1 mutants. Overall, our data demonstrates the importance of PRY-1 and its interactions with the WNT asymmetry pathway components for the regulation of miRNAs (and their targets) during asymmetric cell division. Since the WNT pathway and miRNAs are conserved in eukaryotes, similar interactions with Axin family members may control stem cell division in other systems.

\section{Results}

We observed that pry-1 mutant worms have a weaker cuticle (Fig. 2a) and abnormal alae that frequently includes 

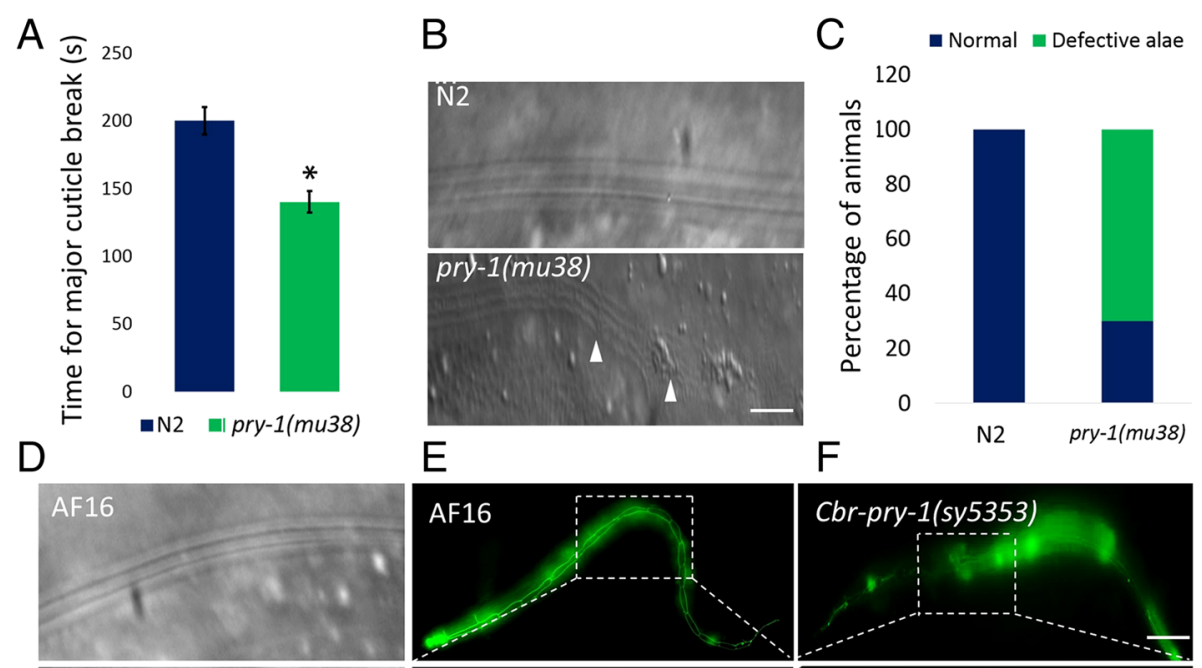

\section{$\mathrm{F}$}

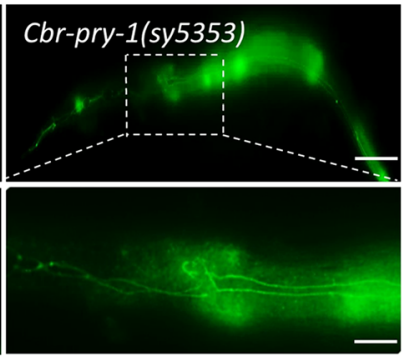

G
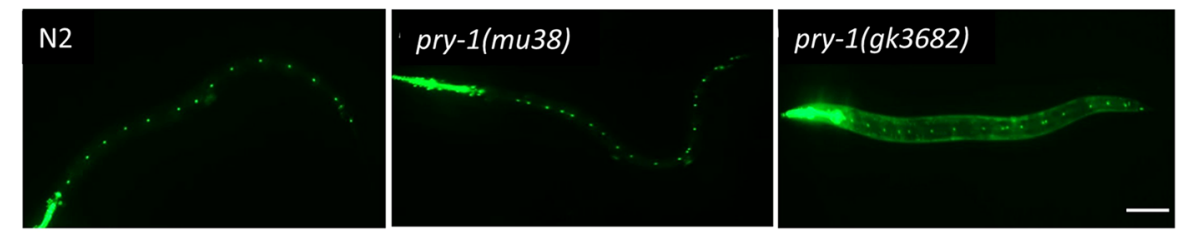

$\mathrm{H}$

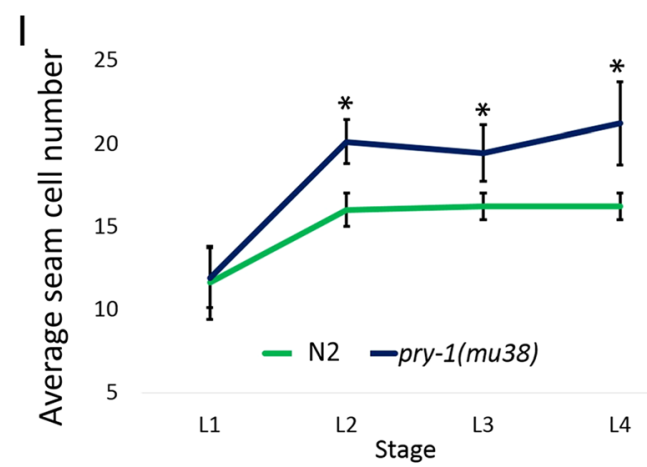

Fig. 2 pry-1 mutants display seam cell defects in C. elegans and C. briggsae. Mutations in pry-1 lead to somatic defects. (a) Cuticle is weaker as demonstrated by the cuticle break assay. (b, c) pry-1 mutants having defective alae formation. (b) Control N2 animals have distinct rows of alae. pry1 (mu38) mutants have defect with frequent breaks (see arrow). (c) Quantification of alae defect in control N2 and pry-1(mu38) animals. (d-f) Heterochronic phenotypes in C. briggsae AF16 and Cbr-pry-1 mutants. (d) Alae defects are visible in Cbr-pry-1 (sy5353). (e, f) Seam cells in control AF16 (e) and Cbr-pry1(sy5353) (f) are visualized by adherens-junction-associated marker Cel-dlg-1p::GFP. Cbr-pry-1(sy5353) animals show defects in cell fusion (scale bar $0.1 \mathrm{~mm}$ ). Boxed areas, marked by dotted lines, have been enlarged in the second row. Scale bars in $\mathbf{b}, \mathbf{d}-\mathbf{f}$ are $0.01 \mathrm{~mm}$. (g) Both the pry-1(mu38) and pry-1(gk3682) animals show increased seam cell numbers compared to control N2 (scale bar $0.1 \mathrm{~mm}$ ). (h) Each control animal has exactly 16 seam cells, whereas an average of 21 and 19 cells are found in pry-1(mu38) and pry-1 (gk3682) mutants, respectively. (i) pry-1(mu38) animals show increased seam cell number by the end of the $\mathrm{L} 2$ stage. In panels $\mathbf{a}, \mathbf{h}$, and $\mathbf{i}$, each data point represents the mean of at least two replicates (each batch with 30 or more worms) and error bar represents the STD. Student's $t$-test was used to determine the statistical significance: ${ }^{*} p<0.05$

gaps (Fig. 2b, c). The phenotypic analysis of C. briggsae pry1 mutants, Cbr-pry-1(sy5353) [24], revealed similar gaps in alae as well as defective seam cell morphologies (Fig. 2d-f). Given these hypodermis-associated phenotypes, we investigated the role of pry-1 in seam cell development. pry-1 mutants exhibit an increased number of seam cells In C. elegans, seam cells divide asymmetrically at each larval stage to produce two daughter cells, one of which fuses with the hypodermal syncytium, while the other retains the seam cell fate (Fig. 1). The L2 stage is unique 
because it also includes a symmetric division that causes an increase in the number of seam cells (Fig. 1). We found that the pry-1(mu38) mutants have an average of approximately five extra seam cells (Fig. 2g, h), consistent with a previously report [19]. A similar phenotype was also observed in pry1 (gk3682), a new CRISPR/Cas9-induced mutant strain (provided by Don Moerman's lab) (Fig. 2g, h; Additional file 1: Figure S1). A stage-specific analysis conducted using scm:: GFP and ajm-1::GFP markers [26] revealed a higher number of seam cells in pry-1 mutants by the end of the L2 stage, likely due to an increase in symmetric cell divisions (Fig. 2h, i). Together, these findings suggest that pry-1 appears to play a role in L2-specific seam cell division.

\section{Heterochronic miRNA expression is altered in pry-1 mutants}

As described above, seam cell asymmetry is mediated by two interacting pathways. While heterochronic genes, such as members of the lin-4 and let-7 miRNA families, control cell division, the WNT asymmetry pathway plays a role in the specification of anterior/posterior daughter cell fates. To evaluate the involvement of miRNAs in pry-1-mediated seam cell development, we performed RNA-Seq experiment in L1-stage animals. The results revealed six DE miRNAs in the pry-1(mu38) mutants. Additionally, 61 novel miRNAs were recovered in the $C$. elegans reference sample (N2) (see Methods and Additional files 2-4: Table S1, S2, S3) that serve as a resource to further investigate the miRNA biology in worms.

Of the six DE miRNAs, five (lin-4, miR-48, miR-84, $m i R-237$, and $m i R-241)$ were upregulated and one (miR246) was downregulated (Fig. 3a, b). Notably, all of these except miR-246 are known to be involved in both heterochronic development and a range of other processes $[8,9$, 27,28 , a fact that was further supported by our tissueenrichment analysis (Additional file 5: Figure S2). We next performed quantitative real-time PCR (qRT-PCR) validations which revealed similar, if comparatively lower (322-fold versus 2-12-fold in RNA-Seq and qRT-PCR, respectively), DE trends (Fig. 3B, C). Moreover, the C. briggsae orthologs of DE miRNAs were found to be likewise altered in Cbr-pry-1(sy5353) animals (Fig. 3d).

To elucidate whether pry-1-mediated miRNA regulation is stage-specific, we examined miRNA transcript levels by qRT-PCR in adult nematodes. While the expression of miRNAs was either unchanged or downregulated in pry-1(mu38) (Additional file 6: Figure S3A), the pattern was different in Cbr-pry-1(sy5353) animals, i.e., three miRNA orthologs were up, two were down, and one was unchanged (Additional file 6: Figure S3B). We also used the existing C. elegans miRNA::GFP transgenic strains [29] to determine changes in miRNA expression in pry-1(mu38) animals and confirmed the qRT-PCR results (Additional file 7: Figure S4). Overall, the dissimilar expression trends of the analyzed miRNAs in adults from L1-stage nematodes suggests that the pry1 -miRNA network is likely temporally regulated.

$m i R-246$, which a previous study showed to be involved in aging, oxidative stress, and thermo-sensation $[30,31]$, was the only miRNA to be downregulated in the pry-1(mu38) nematodes. Although no role in heterochronic development has yet been reported for $m i R-246$, we herein demonstrate that $m i R-246(n 4636)$ adults exhibit alae defects (Fig. 3e). Conversely, scm::GFP and ajm-1::GFP reporter-based expression analyses of the miR-246(n4636) adults did not reveal any significant change to the seam cells (Fig. 3f). This finding was supported by the results of the tissue-enrichment analysis (Additional file 5: Fig. S2). Interestingly, a hypodermal cell marker, $d p y-7:: H 1$-wcherry, revealed that the number of hypodermal cells was reduced in the mutant compared to control worms $(45.1 \pm 1.7, n=20$ and $51.9 \pm 1.6$, $\mathrm{n}=20$, respectively; also see Additional file 8: Figure S5). Further study is needed to determine the exact fate of these hypodermal cells in miR-246 mutants.

\section{Many predicted gene targets of the mis-regulated heterochronic miRNAs are differentially expressed in the pry-1(mu38) mRNA transcriptome}

miRNAs mediate the degradation or translational inhibition of their target mRNAs via binding between their seed sequence and an miRNA response element (MRE) in the 3' untranslated region of their target. Therefore, we searched for miRNA targets using TargetScan online program (http:// www.targetscan.org/vert_72/), and resultantly identified 453 unique targets. The gene ontology (GO) analysis revealed that these predicted miRNA targets were predominantly associated with processes such as the regulation of heterochrony (29-fold enrichment), the positive regulation of nematode larval development (8-fold enrichment), the molting cycle (5-fold enrichment), and collagen and cuticulinbased cuticle development (7-fold enrichment) (Fig. 4a).

A comparison of the predicted miRNA target genes with our recently published pry-1(mu38) mRNA transcriptome [25] revealed a significant overlap (111 genes, Representation factor: 1.6, hyp.geo $p<7.862$ e-08) (Fig. $4 \mathrm{~b})$. Furthermore, a tissue-enrichment analysis showed that this subset of overlapping genes is frequently associated with the hypodermal syncytium (i.e. the third-most enriched subset) (Fig. 4c). Together, these findings suggest that PRY-1 is likely necessary for normal miRNA expression during seam cell development.

\section{Knockdowns of WNT asymmetric pathway components affect both the pry-1(mu38) phenotype and miRNA expression}

We induced RNAi-mediated silencing to examine interactions between pry-1 and WNT asymmetry pathway components 

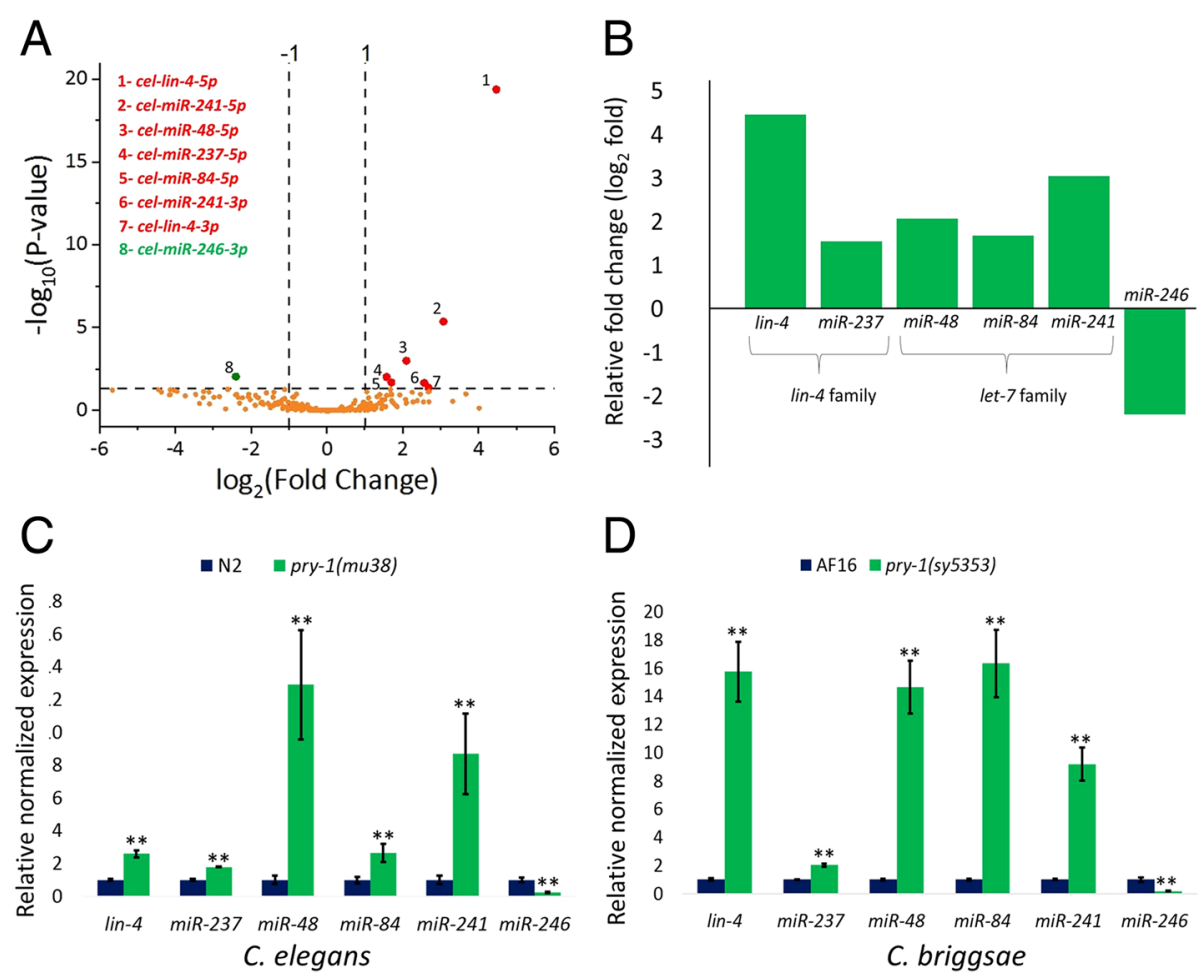

$\mathrm{E}$

$\mathrm{F}$
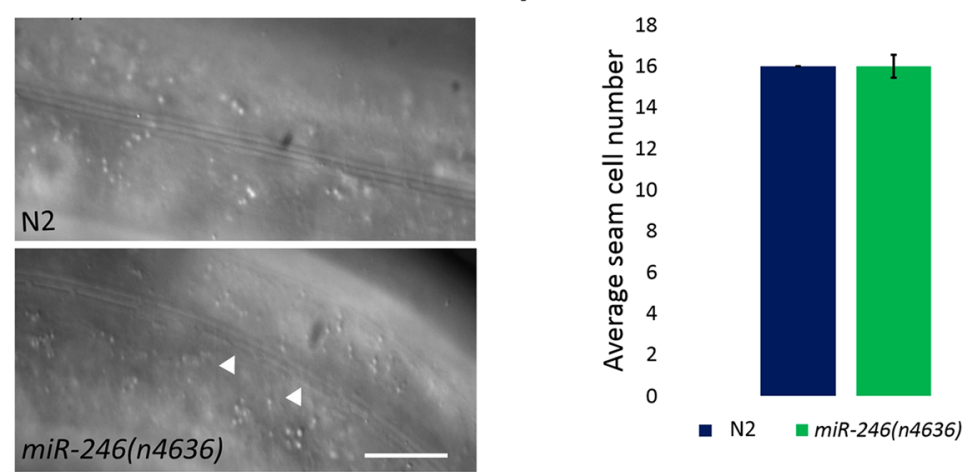

Fig. 3 pry-1 is necessary for normal miRNA expression in both C. elegans and C. briggsae. (a) Volcano plot of differentially expressed miRNA genes in pry-1 (mu38). Red and green dots mark significantly altered upregulated and downregulated transcripts, respectively, with a $p$-adj value of $<$ 0.05. Orange dots mark transcripts that are not significantly altered ( $p$-adj > 0.05). (b) Data showing $\log _{2}$ fold expression of miRNAs from RNA-Seq analysis. (c, d) qRT-PCR analysis of miRNAs in Cel-pry-1(mu38) and Cbr-pry-1(sy5353) animals at the L1 stage showed upregulation of all but miR246. Each data point represents the mean of two replicates and error bar represents the SEM, ${ }^{* *} p<0.01$. (e, $\mathbf{f}$ ) miR-246(n4636) mutants exhibit alae defect in the form of gaps (scale bar $0.01 \mathrm{~mm}$ ) (e) but show no change in the number of seam cells (f). Each data point represents the mean of at least two replicates (each batch with at least 20 worms) and error bar represents the STD

during seam cell development. The fates of seam cell daughters are specified by the nuclear levels of POP-1 that are high in the anterior cell (hypodermal fate) and low in the posterior cell (seam cell fate) [19, 32] (Fig. 5a). The results of our experiments revealed that knockdowns of wrm-1 or lit-1 suppresses the pry1(mu38) seam cell phenotype (Fig. 5b), likely because PRY-1 promotes asymmetric division by negatively regulating both of these factors in anterior daughter cells (Fig. 5a). This is consistent with PRY-1 being localized to the anterior cortex of dividing seam cells [23]. A similar genetic interaction between pry-1,wrm-1, and lit-1 was previously reported to occur during the asymmetric division of embryonic EMS cells [33].

In contrast to wrm-1 and lit-1, pop-1 RNAi exacerbated the pry-1(mu38) phenotype, resulting in a significant increase in the number of seam cells $(35.9 \pm 10.8$ in pry-1(mu38); pop-1(RNAi), compared to $20.8 \pm 2.1$ in pry-1(mu38), and $23.5 \pm 5.7$ in pop-1(RNAi)) (Fig. 5b, c). We also examined nuclear POP-1 asymmetry following 


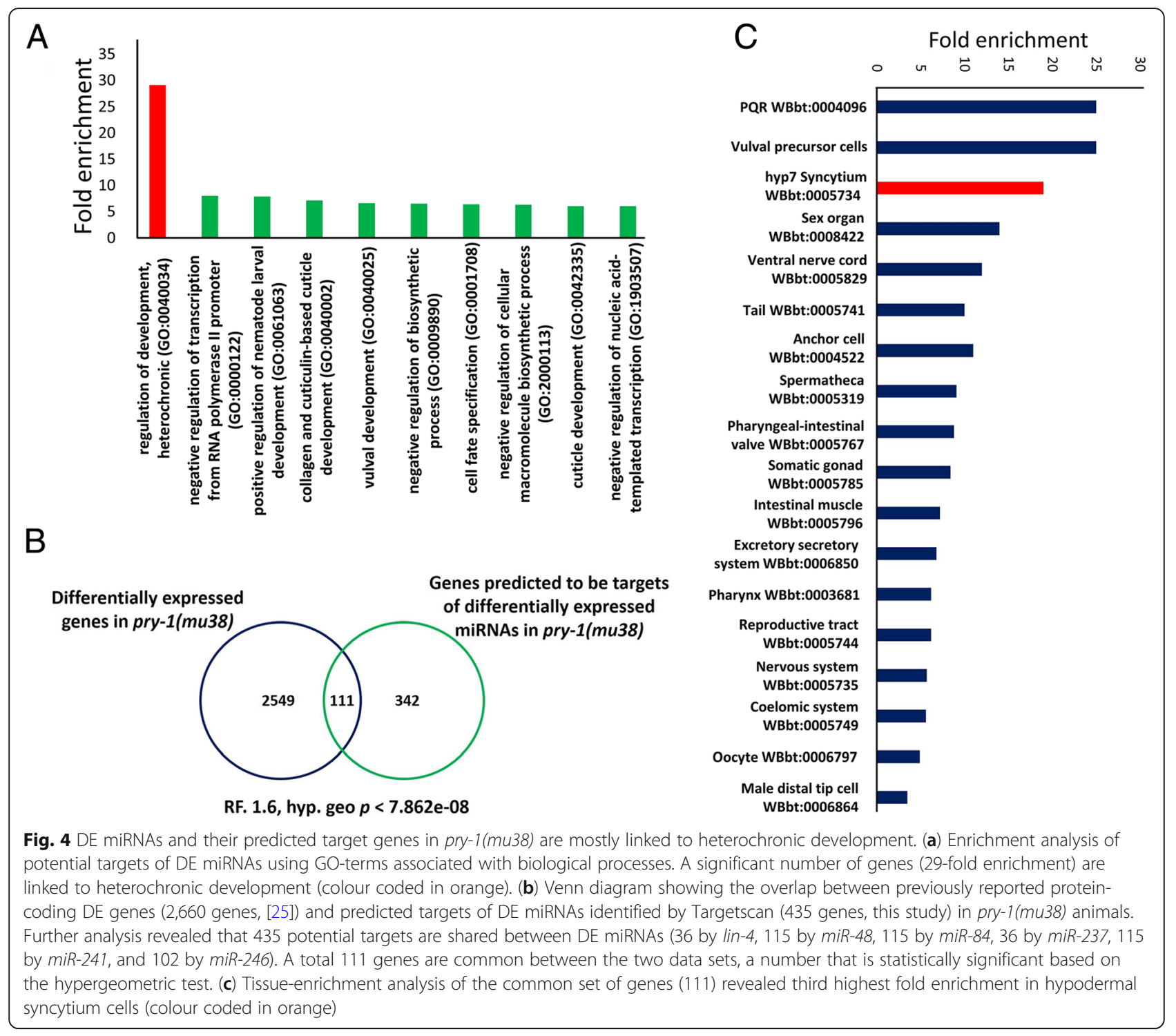

RNAi knockdown of pry-1 and found that it was disrupted (Fig. 5d). These results agree with nuclear POP-1 levels being differentially regulated by WRM-1 and LIT1 to be higher in the anterior, and lower in the posterior daughter cell [19, 32] (Fig. 5a) Together, our findings support that pop-1 likely limits the number of seam cells that are produced by promoting the asymmetric division of their precursors. Since the asymmetric localization of WRM-1, LIT-1, and POP-1 are known to mediate fate specification in presumptive seam cells, our results suggest that PRY-1 likely facilitates the maintenance of these asymmetric expression patterns.

Given that the nuclear POP-1 and SYS-1 ratio determines the fate of daughter cells and SYS-1 localization is disrupted in animals lacking PRY-1 function [34], we also examined the effect of sys-1 knockdown. The results of this analysis showed no effect on seam cell division in pry-1(mu38) animals (Fig. 5b). We likewise knocked down another $\beta$-Catenin family member, bar-1, in the mutant strain and found that doing so did not alter the number of seam cells (Fig. 5b). Overall, the data support the possibility that $\beta$-Catenin family members are functionally redundant during seam cell division, consistent with previous studies [19], although do not exclude the possibility that PRY-1 role in seam cells is independent of BAR-1 and SYS-1.

To examine whether WRM-1, LIT-1, and POP-1 asymmetries affect miRNA expression during seam cell division, we next quantified miRNA levels in animals in which POP-1 function was compromised. As in pry1(mu38) mutants, the expression levels of lin-4, miR-48, $m i R-84, m i R-237$, and $m i R-241$, were found to be significantly upregulated in both pop-1(hu9) and pop-1(RNAi) worms; however, no change to miR-246 expression was 

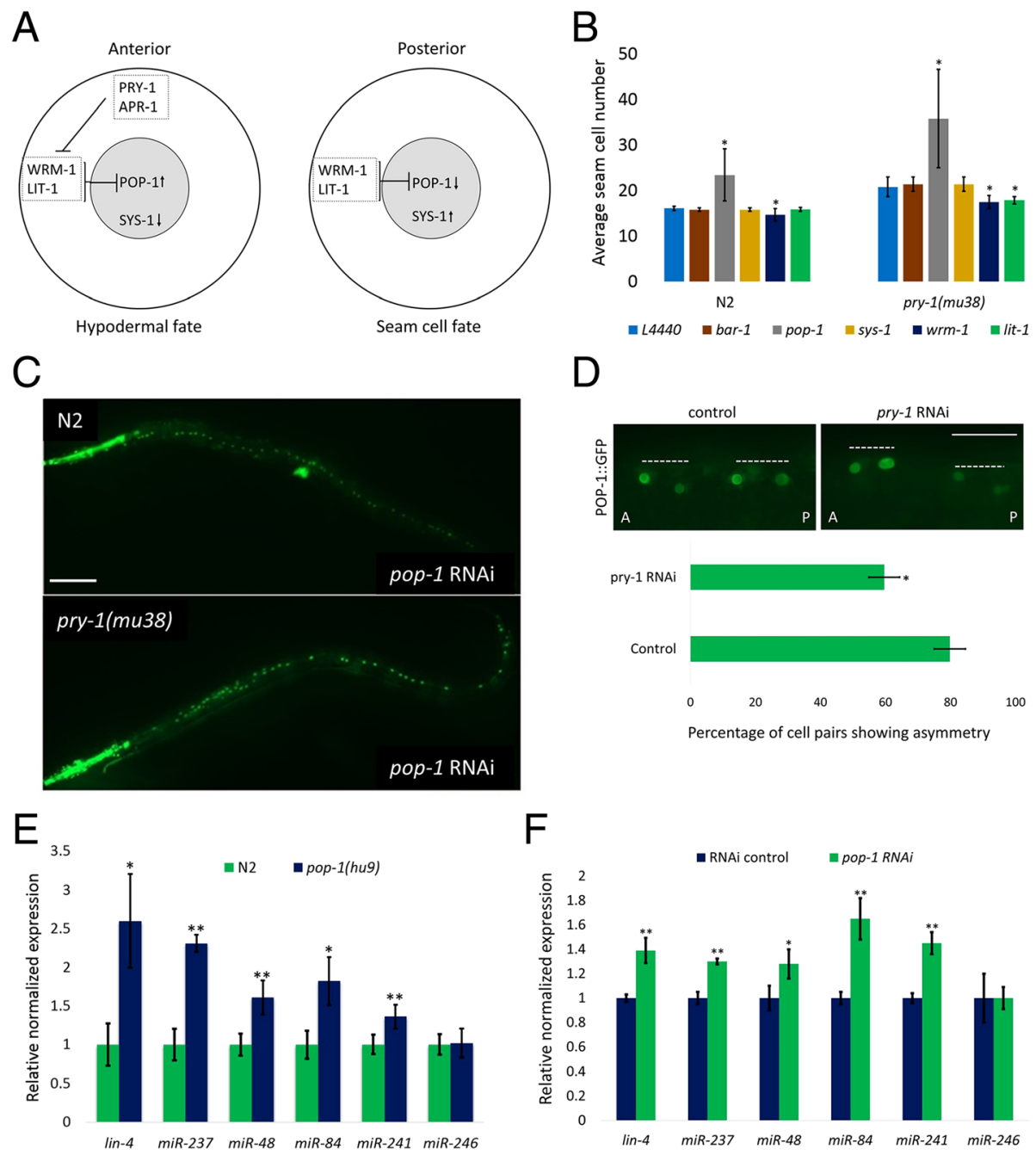

Fig. 5 WNT asymmetry pathway components WRM-1, LIT-1, and POP-1 are important for PRY-1-mediated expression of miRNAs and seam cell development. (a) Schematic diagram showing the involvement of PRY-1 and other WNT asymmetry pathway components in the specification of seam cell fates. The levels of nuclear POP-1 are high in the anterior cell but low in the posterior cell. (b) RNAi knockdowns of bar-1, pop-1, wrm-1, sys-1 and lit-1 in control N2 and pry-1(mu38) animals. Each data point represents the mean of at least two batches (each batch with at least 30 worms) and error bar represents the STD. Student's $t$-test was used to determine the statistical significance: ${ }^{*} p<0.05$ (compared to L4440 control). (c) Representative images of control N2 and pry-1(mu38) animals following pop-1 RNAi. The numbers of seam cells are increased in both cases. The phenotype is particularly enhanced in pry-1 mutants (scale bar $0.1 \mathrm{~mm}$ ). (d) Representative images of two adjacent POP-1::GFP fluorescing cell pairs from V1-V5 seam cell lineages following control and pry-1 RNAi treatments (A-anterior, P- posterior, each dotted line marks a cell pair, scale bar $20 \mu \mathrm{m})$. POP-1 asymmetry is disrupted after pry-1 RNAi, resulting in fewer cell pairs having asymmetric localisation of POP-1::GFP in their nuclei. (e, f) qRT-PCR analysis of miRNAs in pop-1(hu9) and pop-1(RNAi) worms at the L1 stage. Similar to pry-1(mu38), all miRNAs, except miR-246, are overexpressed in both pop-1(hu9) (e) and pop-1(RNAi) (f) animals. Each data point represents the mean of two replicates and error bar represents the SEM, ${ }^{*} p<0.05,{ }^{* *} p<0.01$

observed (Fig. 5e, f). Furthermore, the bioinformatic analysis revealed multiple TCF/LEF consensus binding sites (SCTTTGATS; S = G/C) $[35,36]$ in the $5^{\prime}$ regulatory region of each of these miRNAs, except for miR-246 where a single site is found near the transcriptional start site (See Methods, Additional file 9: Figure S6), suggesting that their transcription may be inhibited by POP-1. Together, these data allow us to conclude that miRNAs act downstream of POP-1, which agrees with a previous model [18], and also suggest that PRY-1 may interact with WRM-1, LIT-1, and POP-1 to negatively regulate the expression of heterochronic miRNAs during seam cell development.

HBL-1 and LIN-28 act genetically downstream of PRY-1 and POP-1 signaling during asymmetric seam cell division To further examine the role of pry-1 in miRNAmediated heterochronic development, we focused on three known miRNA targets, hbl-1, lin-14, and lin-28. Previous studies have shown that lin-14 and lin-28 are 
targeted by lin- 4 [3, 5, 37, 38], whereas $h b l-1$ and $l i n-28$ are targeted by another lin-4 family member $m i R-237$ [8, $39,40]$ as well as let-7 family members $m i R-48, m i R-84$, and $m i R-241[9,17]$.

The qRT-PCR analysis showed that while lin-14 expression levels were unchanged, $h b l-1$ and $l i n-28$ were significantly downregulated in L1-stage pry-1(mu38) animals (Fig. 6a). Together with results described in previous sections, this observation allows us to propose that pry-1 may function upstream of miRNAs to promote expression of $h b l-1$ and $l i n-28$. To further examine the regulatory network of pry-1, RNAi was carried out. The results revealed that while lin-14 and $h b l-1$ RNAi had no marked impact on seam cells, lin-28 RNAi caused a significant reduction in the seam cell number in both pry-1(mu38) and control animals (Fig. 6b).

\section{Discussion}

In this paper we describe a genetic pathway of PRY-1/ Axin signaling in seam cell development. Using a combination of mutant analysis and reporter gene expression we show that PRY-1 is involved in L2-specific seam cell division. To identify the genes regulated by pry-1, we performed whole genome miRNA profiling at the lateL1 stage. The results revealed six DE miRNAs in pry-1 mutants. Five of these, belonging to lin-4 and let-7 families (lin-4, miR-48, miR-84, miR-237, and miR-241), were upregulated whereas $m i R-246$ was the only miRNA that was downregulated. A similar trend was also observed in C. briggsae pry-1 mutants suggesting that pry-1 plays a conserved role in miRNA regulation in Caenorhabditis nematodes.

Three of the overexpressed miRNAs in pry-1 mutants, namely $m i R-48$, miR-84, and miR-241 (let-7 family members), are known to redundantly control the L2-L3 larval-stage transition [9]. While C. elegans nematodes carrying a mutation in any one of these three miRNAs have been shown to exhibit a normal phenotype, miR48/miR-84 double mutants display retarded molting and a higher number of seam cells as a result of reiterated symmetric divisions during the L2 stage [9]. This seam cell phenotype is further exacerbated in $m i R-48 / m i R-84 /$ miR-241 triple mutants [9], while conversely, miR-48overexpression mutants were shown to exhibit a reduced number of seam cells due to 'skipping' of L2-specific symmetric divisions [41]. The other two miRNAs upregulated in the pry-1(mu38) mutants are lin-4 and miR237 (lin-4 family members [42]). A previous study showed that, although a miR-237-knock-out mutant does not directly incur a heterochronic defect, it enhances the seam cell phenotype exhibited by lin-4(e912); lin-14(n179ts) double mutant animals [8].

Unlike lin-4 and let-7 family of DE miRNAs, miR-246 is not known to play a role in heterochronic development although it is involved in other processes [30, 31]. Consistent with previous studies, our analysis of $m i R$ 246 mutants did not reveal any changes in the number of seam cells. Thus, pry-1-mediated miR-246 regulation may participate in other biological events.

The fact that all but one DE miRNAs in pry-1 mutants are involved in heterochronic pathway suggests an important role of pry-1 in this developmental process. This was further strengthened by our data showing a high enrichment for miRNA predicted target genes associated with GO-term processes such as regulation of heterochrony. Moreover, we observed a significant overlap between DE miRNA predicted targets and pry-1(mu38) mRNA transcriptome [25] that included many genes expressed in hypodermal syncytium.

To understand the regulation of miRNAs by pry-1 we studied the involvement of WNT asymmetry pathway components using an RNAi approach. It was shown
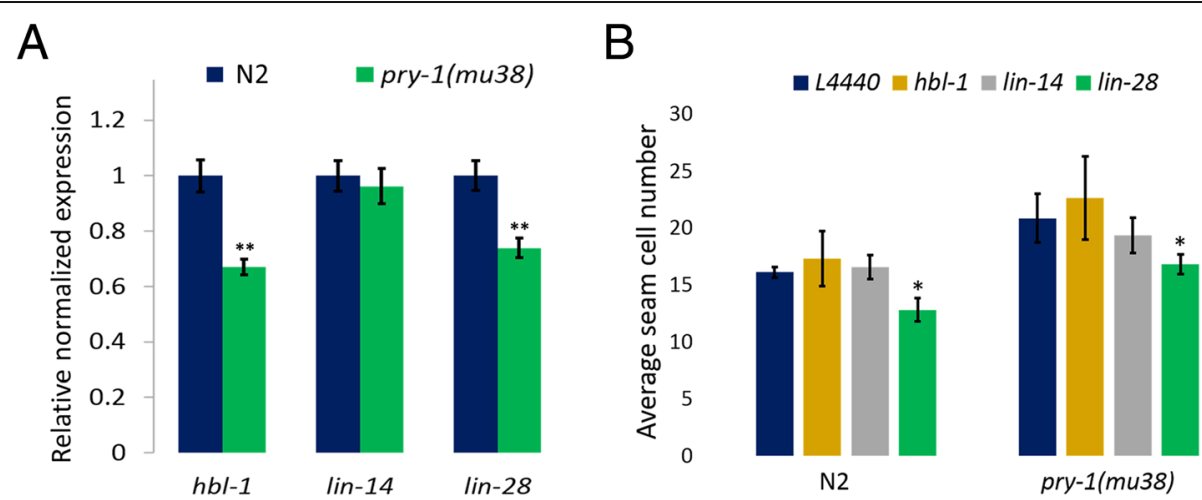

Fig. 6 Analysis of heterochronic genes in pry-1-mediated seam cell development. (a) qRT-PCR experiments at the L1 stage showed that $h b l-1$ and lin-28 are significantly downregulated in pry-1(mu38) animals. Each data point represents the mean of two replicates and error bar represents the SEM, ${ }^{* *} p<0.01$. (b) RNAi KD of lin-28 in pry-1(mu38) mutants rescued the seam cell defect. Each data point represents the mean of three batches (each batch with at least 30 worms) and error bar represents the STD. Student's $t$-test was used to determine the statistical significance: * ${ }^{*}<0.05$ (compared to L4440 control) 
earlier that in the absence of PRY-1, localizations of WRM-1, LIT-1, and SYS-1 are disrupted [23, 34]. As expected, the examination of seam cell phenotypes in pry1(mu38) animals following RNAi knockdowns of these genes revealed that wrm-1 and lit-1 are necessary for pry-1 function. Thus, PRY-1 may affect seam cell number by localizing in the anterior cortex of dividing seam cells [23] and thereby lowering WRM-1 and LIT-1mediated nuclear POP-1 levels in anterior daughters. This plausible explanation is supported by our finding that POP-1 asymmetry is affected in animals with reduced PRY-1 function. To test this further, we examined miRNA expression in pop-1(hu9) and pop-1(RNAi) worms. As expected, all five (lin-4, miR-48, miR-84, $m i R-237$, and $m i R-241)$ were found to be overexpressed. Moreover, multiple TCF/LEF binding sites were detected in the $5^{\prime}$ regulatory regions of miRNAs. Together, these findings raise the possibility of POP-1 acting as a transcriptional regulator of miRNAs.

We thus propose a model summarizing our findings (Fig. 7), in which PRY-1 acts upstream of WRM-1, LIT1, POP-1, and DE miRNAs (except miR-246). Since the miRNAs regulate the expression of protein-coding genes during heterochronic development, we tested three of their known targets $h b l-1$, lin-14, and lin-28. The results of our experiments suggested that $h b l-1$ and lin-28 act downstream of pry-1; however, only lin-28 appears to function in pry-1-mediated asymmetric cell division. The

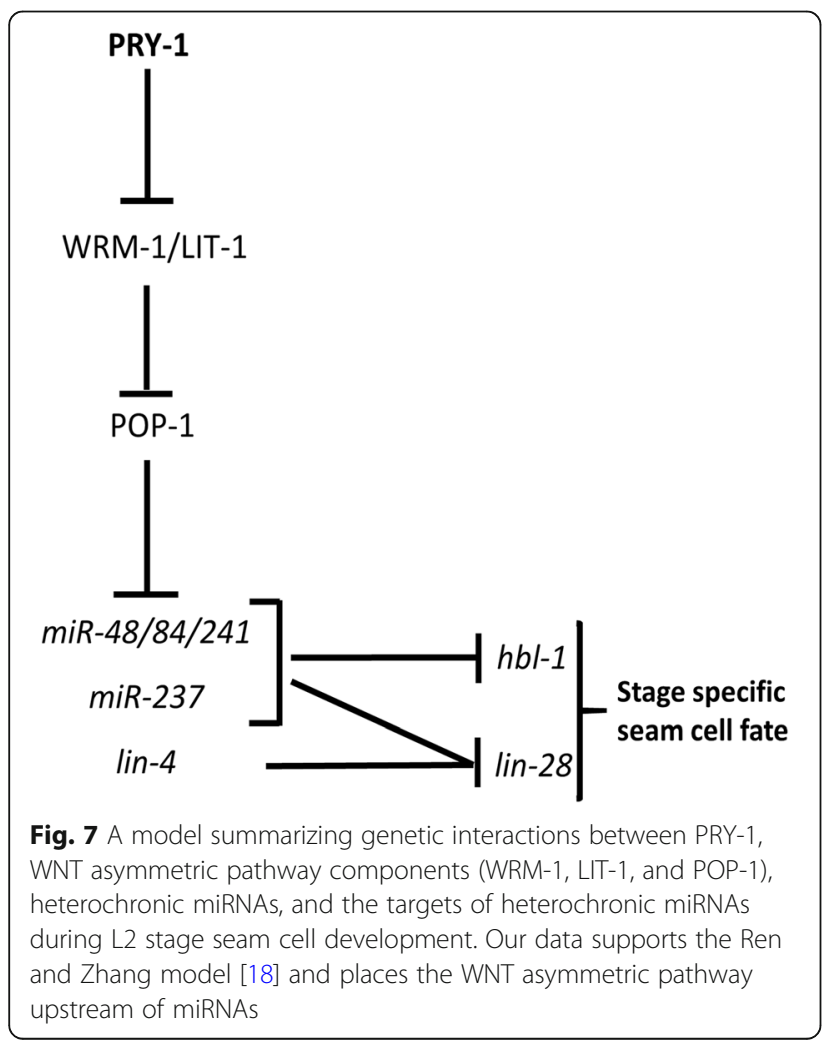

above genetic interactions are consistent with earlier findings where lin-28 was shown to act downstream of the WNT asymmetry pathway components and included in a network consisting of lin-4 and let-7-family of miRNAs and their targets during seam cell development [17, 18]. Our model is unique in that it places PRY-1 upstream of WRM-1, LIT-1, and POP-1-mediated miRNA transcriptional network.

\section{Conclusions}

Overall, the data presented in this paper demonstrate the important role of PRY-1/Axin in the regulation of miRNAs and their heterochronic gene targets in a pathway that involves WNT asymmetry pathway components WRM-1, LIT-1, and POP-1 during seam cell development. Furthermore, since seam cell defects are also exhibited by C. briggsae pry-1 mutants, and that Cbr-pry-1 is necessary for the normal expression of these miRNA orthologs, our work has revealed that the role of pry-1 in seam cell development is conserved amongst nematodes.

\section{Methods}

Strains, culture condition, and RNAi

Nematodes were grown on standard NG-agar culture plates seeded with $E$. coli bacteria (OP50) [43]. Cultures were maintained at $20^{\circ} \mathrm{C}$. Strains used in the study are listed in a supplementary table (Additional file 10: Table S4). RNAimediated gene silencing was performed using a protocol previously published by our laboratory [24].

\section{Microscopy}

Nematodes were mounted on a glass slide containing 2\% agarose and $0.02 \mathrm{M} \mathrm{NaN}_{3}$ and observed using an Axiovision Zeiss microscope. Seam cell nuclei were counted, and adult lateral alae were scored using Nomarski differential interference contrast and epifluorescence optics. Images were acquired using NIS Element software (Nikon, USA) with a Hamamastu Camera that was mounted on a Nikon 80i upright microscope.

\section{Analysis of seam cell division}

The fates of daughter seam cells were determined in $C$. elegans using scm::GFP and ajm-1::GFP markers, and in $C$. briggsae using the seam cell adherens-junction marker, Cel-dlg-1::GFP. After $8 \mathrm{~h}$ of feeding, nematodes expressing GFP that had completed the first larval seam cell division, and that had 10 seam cells per side, were chosen for analysis. Seam cell divisions were monitored at approximately $6 \mathrm{~h}$ intervals until the late $\mathrm{L} 4$ stage, when divisions ceased.

\section{Cuticle integrity assay}

A solution containing $1 \%$ hypochlorite and $0.25 \mathrm{M}$ $\mathrm{NaOH}$ was prepared as previously reported [44], and aliquoted $(500 \mu \mathrm{l})$ into a 48 -well plate. Individual gravid 
adult worms were transferred to each well, and the plate was agitated at $30 \mathrm{~s}$ intervals. The time to the first major cuticle break was recorded by direct observation using a dissecting stereoscope (SMZ 645; Nikon Corporation, Japan).

\section{Molecular biology and bioinformatics}

For qRT-PCR experiments, synchronous cultures were prepared by bleaching gravid hermaphrodites as described previously [45] except that eggs were allowed to hatch on the NG-agar plates. The bleaching process was repeated. The eggs were finally transferred onto plates and grown till the desired stage. Because pry-1 mutants grow slower than controls, RNA was extracted from age-matched animals. The L1 larvae were grown till $16 \mathrm{~h}$ (N2/AF16) and $18 \mathrm{~h}$ (pry-1 mutants) whereas adults were incubated for $52 \mathrm{~h}$ (N2/AF16) and $58 \mathrm{~h}$ (pry-1 mutant). Total RNA was extracted from animals using the trizol reagent (Catalog Number T9424, Sigma-Aldrich, Canada), according to the manufacturer's instructions. cDNAs for protein-encoding genes and miRNAs were synthesized using oligo-dT and specific stem-loop primers, respectively (Additional file 11: Table S5), and by using the qScript cDNA synthesis kit (Catalog Number 95047-025, Quantabio, Canada) according to manufacturer's instructions.

qRT-PCR was performed (in triplicate) in the Bio-Rad cycler CFX 96 using appropriate primers (Additional file 11: Table S5), and SensiFAST SYBR Green Kit (Catalog Number BIO-98005, BIOLINE, USA), according to the manufacturer's instructions. The expression levels of miRNAs and protein-coding genes were normalized to those of $m i R-2$ and $p m p-3$, respectively. Ct and $p$ values were calculated using CFX manager software (Bio-Rad, Canada).

A lin-28 RNAi plasmid was constructed by inserting $2949 \mathrm{bp}$ of the lin-28 coding sequence into the L4440 vector. A DNA fragment was obtained via PCR using the listed primers (Additional file 11: Table S5), under the specified PCR conditions.

To identify TCF/LEF family binding sites in the $5^{\prime}$ upstream genomic region of the miRNAs, MatInspector software (https://www.genomatix.de/) was used with default settings.

\section{RNA-Seq experiment}

The steps for miRNA RNA-Seq in pry-1(mu38) mutants were similar to mRNA RNA-Seq that we reported earlier [25]. The pry-1 miRNA transcriptome profile can be found in the GEO archive with accession number GSE130039. Synchronized L1 worms were harvested by two successive bleaching to obtain a homogenous population and total RNA was isolated. Small RNA sequencing libraries were prepared, and samples were analysed using the Genome Analyzer IIx platform (Illumina Inc.,
USA) at the McGill University Genome Quebec sequencing facility.

A total of 36,656,022 reads of small RNAs (15-25 nt) were generated from the four samples of $C$. elegans examined, of which 10,453,527 sequences aligned perfectly to the C. elegans genome. Overall, we detected perfect matches to the precursor forms of 161 out of the 250 miRNAs annotated in miRBase (http://www.mirbase.org) WBcel235 for C. elegans. DE analysis of the known miRNAs led to identification of eight miRNAs that were altered in pry-1 mutants, of which two (miR-353 and miR$2208 a$ ) were excluded due to false predictions (Additional file 2: Table S1).

Although previous studies have reported miRNAs in the C. elegans genome (e.g., see [42, 46], due to increased depth of our sequencing data we expected to uncover additional new candidates. After eliminating rRNA, tRNA, and ncRNA reads, the remaining unannotated reads were processed for novel miRNA discovery, as discussed below. We focused on the 30,300 nonredundant un-annotated reads that aligned to the $C$. elegans genome in control $\mathrm{N} 2$ and pry-1(mu38) animals. To discover novel miRNAs, the miRNA discovery package miRDeep2 (https://www.mdc-berlin.de/n-rajewsky\#t-data,software\&resources) was used. The analysis predicted a total of 243 miRNAs using read count cutoff of 5-fold (or 187 with cut-off of 10-fold) (Additional file 3: Table S2). We then used an additional criterion to further examine these candidates, i.e., a higher miRDeep Score $(>10)$. This led to the identification of 64 novel miRNAs at the 5 -fold read count cut-off (or 61 when the read count was set at 10-fold) (Additional file 3-4: Table S2, S3). The authenticity of these novel miRNAs was tested by RNAfold, which confirmed that these produce miRNA stem loop structure [47].

\section{Statistical analyses}

Statistics were performed by two-tailed student's $t$ test after testing for equal distribution of the data and equal variances within the data set. The $p$ values of 0.05 and less were considered statistically significant. The data are presented as either mean \pm standard deviation of the mean (STD) or mean \pm standard error of mean (SEM). Graphs were prepared using Microsoft Excel. Hypergeometric probability related tests were done using an online program (http://nemates.org/MA/progs/overlap_stats.html).

\section{Additional files}

Additional file 1: Figure S1. C. elegans pry-1 open reading frame showing the region affected by gk3682 mutation. The exons and introns are indicated by boxes and lines, respectively. The translational start and stop sites are marked. The sequence deleted in gk3682 allele (738 bp) is shown by a rectangle. As part of the CRISPR editing process, the excised 
portion is replaced by a 5419 bp myo-2::GFP containing cassette. The allele and sequencing data were kindly provided by Dr. Moerman's lab. (TIF $157 \mathrm{~kb}$ )

Additional file 2: Table S1. A list of miRNAs in pry-1 (mu38) animals. The file contains two spreadsheets that list all miRNAs identified by RNA-Seq experiment (used for volcano plot, see Fig. 3a) and DE miRNAs. (XLSX 25 kb)

Additional file 3: Table S2. Total number of novel miRNAs in C. elegans. The table shows the number of predicated miRNAs based on different miRDeep scores and read count cut-offs. (DOCX $13 \mathrm{~kb}$ )

Additional file 4: Table S3. A list of novel miRNAs in C. elegans. The IDs of miRNAs are based on the sequential numbering of unique reads in our analysis. For each novel miRNA, the table lists the miRDeep score, read count, chromosomal location, and the sequence. (XLSX $380 \mathrm{~kb}$ )

Additional file 5: Figure S2. Tissue-enrichment analysis of DE miRNAs. The analysis was done using the miRNA discovery tool miRDeep2 (see the RNA-Seq section in Methods). For each miRNA, colored areas represent tissue-specific expression. (TIF $9730 \mathrm{~kb}$ )

Additional file 6: Figure S3. Adult stage qRT-PCR analysis of heterochronic miRNAs in C. elegans and C. briggsae pry-1 mutants. (A) pry-1(mu38) adults show differences in the pattern of miRNA expression compared to the L1 stage. All miRNAs, except lin-4 and miR-48, are downregulated. (B) Cbr-pry1(sy5353) adults show altered expression of miR-246, miR-48 and miR-84. Each data point represents the mean of two replicates and error bar represents the SEM, ${ }^{*} p<0.05,{ }^{* *} p<0.01$ (TIF $3950 \mathrm{~kb}$ )

Additional file 7: Figure S4. miRNA expression analysis in pry-1 (mu38) adults using a GFP reporter. (A) Representative images of PmiR-48::GFP, PmiR-84::GFP, PmiR-241::GFP, and PmiR-246::GFP reporters in control N2 and pry-1(mu38) animals. The scale bar is $0.1 \mathrm{~mm}$. (B) Quantification of fluorescence intensity using an arbitrary unit (a.u.) scale. Each data point represents the mean of two replicates (at least 20 animals each) and error bar represents the STD. Student's $t$-test was used to determine the statistical significance: ${ }^{*} p<0.05$. (TIF $7910 \mathrm{~kb}$ )

Additional file 8: Figure S5. Representative images of control N2 and miR-246(n4636) mutants showing hypodermal cells (based on dpy-7::H1wcherry reporter). The mutant animal shows fewer hypodermal cells. (TIF $9910 \mathrm{~kb}$ )

Additional file 9: Figure S6. Line drawings of TCF/LEF putative binding sites in the $5^{\prime}$ upstream regions of miRNA genes (within 1500 bp of transcriptional start site). Each putative binding site is shown by a coloured square box. The numbers in brackets next to boxes show matrix similarity scores. (TIF $7130 \mathrm{~kb}$ )

Additional file 10: Table S4. A list of strains used in this study. (DOCX $33 \mathrm{~kb}$ )

Additional file 11: Table S5. A list of primers used in this study. (DOCX $16 \mathrm{~kb})$

\section{Abbreviations}

DE: Differentially expressed; GO: Gene ontology; miRNAs: microRNAs; MRE: miRNA response element; NLK: NEMO-like kinase; qRT-PCR: quantitative real-time PCR; SEM: Standard error of the mean; STD: Standard deviation; TCF/LEF: T-cell factor/lymphoid enhancer factor

\section{Acknowledgements}

We are grateful to Brian Golding (McMaster University) for providing access to his lab computer server and Don Moerman's lab (University of British Columbia) for generating the pry-1(gk3682) CRISPR/Cas9 allele. Lesley MacNeil and lan Chin-Sang kindly provided comments on a previous draft of the manuscript. Some of the strains were obtained from Caenorhabditis Genetic Center (CGC) which is funded by the NIH Office of Research Infrastructure Programs (P400D010440).

\section{Authors' contributions}

$A M, A R$, and $B G$ designed the study. $A M$ and $A R$ performed experiments. $A M$ and BG wrote the manuscript. BG supervised the project. All authors have read and approved the manuscript.

\section{Funding}

This work was supported by a Discovery grant from the Natural Sciences and Engineering Research Council of Canada (http://www.nserc-crsng.gc.ca) to Bhagwati Gupta. The funder had no role in study design, data collection and analysis, decision to publish, or preparation of the. manuscript.

\section{Availability of data and materials}

The datasets supporting the conclusions of this article are provided in figures, tables, and additional files. miRNA transcriptome data of pry-1 mutants can be found in the NCBI GEO archive database with accession number GSE130039.

\section{Ethics approval and consent to participate}

Not Applicable.

\section{Consent for publication}

Not Applicable.

\section{Competing interests}

The authors declare that they have no competing interests.

\section{Author details}

${ }^{1}$ Department of Biology, McMaster University, ON L8S-4K1, Hamilton, Canada. 2Department of Physics, Harvard University, NW256, 52 Oxford St, Cambridge, MA 02138, USA.

Received: 28 May 2019 Accepted: 4 July 2019

Published online: 15 July 2019

\section{References}

1. Hall DH, Altun ZF. C. elegans Atlas. New York: Cold Spring Harbor Laboratory Press; 2008.

2. Ambros V, Horvitz HR. Heterochronic mutants of the nematode Caenorhabditis elegans. Science. 1984:226(4673):409-16.

3. Lee RC, Feinbaum RL, Ambros V. The C. elegans heterochronic gene lin-4 encodes small RNAs with antisense complementarity to lin-14. Cell. 1993: 75(5):843-54.

4. Wightman $\mathrm{B}, \mathrm{Ha}$ I, Ruvkun G. Posttranscriptional regulation of the heterochronic gene lin-14 by lin-4 mediates temporal pattern formation in C. elegans. Cell. 1993;75(5):855-62.

5. Moss EG, Lee RC, Ambros $\mathrm{V}$. The cold shock domain protein LIN-28 controls developmental timing in C. elegans and is regulated by the lin-4 RNA. Cell. 1997:88(5):637-46

6. Slack FJ, Basson M, Liu Z, Ambros V, Horvitz HR, Ruvkun G. The lin-41 RBCC gene acts in the C. elegans heterochronic pathway between the let-7 regulatory RNA and the LIN-29 transcription factor. Mol Cell. 2000;5(4):659-69.

7. Reinhart BJ, Slack FJ, Basson M, Pasquinelli AE, Bettinger JC, Rougvie AE, Horvitz HR, Ruvkun G. The 21-nucleotide let-7 RNA regulates developmental timing in Caenorhabditis elegans. Nature. 2000:403(6772):901-6.

8. Tsialikas J, Romens MA, Abbott A, Moss EG. Stage-specific timing of the microRNA regulation of lin-28 by the Heterochronic gene lin-14 in Caenorhabditis elegans. Genetics. 2017;205(1):251-62.

9. Abbott AL, Alvarez-Saavedra E, Miska EA, Lau NC, Bartel DP, Horvitz HR, Ambros $\mathrm{V}$. The let-7 MicroRNA family members mir-48, mir-84, and mir-241 function together to regulate developmental timing in Caenorhabditis elegans. Dev Cell. 2005;9(3):403-14.

10. Banerjee D, Slack F. Control of developmental timing by small temporal RNAs: a paradigm for RNA-mediated regulation of gene expression. Bioessays. 2002;24(2):119-29.

11. Fay DS, Stanley HM, Han M, Wood WB. A Caenorhabditis elegans homologue of hunchback is required for late stages of development but not early embryonic patterning. Dev Biol. 1999;205(2):240-53.

12. Hong $Y$, Lee RC, Ambros V. Structure and function analysis of $L I N-14$, a temporal regulator of postembryonic developmental events in Caenorhabditis elegans. Mol Cell Biol. 2000;20(6):2285-95.

13. Ruvkun G, Giusto J. The Caenorhabditis elegans heterochronic gene lin-14 encodes a nuclear protein that forms a temporal developmental switch. Nature. 1989;338(6213):313-9

14. Viswanathan SR, Daley GQ, Gregory RI. Selective blockade of microRNA processing by Lin28. Science. 2008;320(5872):97-100. 
15. Lehrbach NJ, Armisen J, Lightfoot HL, Murfitt KJ, Bugaut A, Balasubramanian S, Miska EA. LIN-28 and the poly(U) polymerase PUP-2 regulate let-7 microRNA processing in Caenorhabditis elegans. Nat Struct Mol Biol. 2009; 16(10):1016-20.

16. Tsialikas J, Romer-Seibert J. LIN28: roles and regulation in development and beyond. Development. 2015;142(14):2397-404

17. Harandi OF, Ambros VR. Control of stem cell self-renewal and differentiation by the heterochronic genes and the cellular asymmetry machinery in Caenorhabditis elegans. Proc Natl Acad Sci U S A. 2015;112(3):E287-96.

18. Ren $\mathrm{H}$, Zhang $\mathrm{H}$. Wht signaling controls temporal identities of seam cells in Caenorhabditis elegans. Dev Biol. 2010;345(2):144-55.

19. Gleason JE, Eisenmann DM. Wht signaling controls the stem cell-like asymmetric division of the epithelial seam cells during C. elegans larval development. Dev Biol. 2010;348(1):58-66.

20. Maduro MF, Lin R, Rothman JH. Dynamics of a developmental switch: recursive intracellular and intranuclear redistribution of Caenorhabditis elegans POP-1 parallels Wnt-inhibited transcriptional repression. Dev Biol. 2002;248(1):128-42.

21. Phillips BT, Kidd AR 3rd, King R, Hardin J, Kimble J. Reciprocal asymmetry of SYS-1/beta-catenin and POP-1/TCF controls asymmetric divisions in Caenorhabditis elegans. Proc Natl Acad Sci U S A. 2007;104(9):3231-6.

22. Shetty P, Lo MC, Robertson SM, Lin R. C. elegans TCF protein, POP-1, converts from repressor to activator as a result of Wnt-induced lowering of nuclear levels. Dev Biol. 2005;285(2):584-92.

23. Mizumoto K, Sawa H. Cortical beta-catenin and APC regulate asymmetric nuclear beta-catenin localization during asymmetric cell division in C. elegans. Dev Cell. 2007;12(2):287-99.

24. Seetharaman A, Cumbo P, Bojanala N, Gupta BP. Conserved mechanism of Wnt signaling function in the specification of vulval precursor fates in $C$. elegans and C. briggsae. Dev Biol. 2010;346(1):128-39.

25. Ranawade A, Mallick A, Gupta BP. PRY-1/Axin signaling regulates lipid metabolism in Caenorhabditis elegans. PLoS One. 2018;13(11):e0206540.

26. Koh K, Rothman JH. ELT-5 and ELT-6 are required continuously to regulate epidermal seam cell differentiation and cell fusion in C. elegans. Development. 2001;128(15):2867-80.

27. Ren Z, Ambros VR. Caenorhabditis elegans microRNAs of the let-7 family act in innate immune response circuits and confer robust developmental timing against pathogen stress. Proc Natl Acad Sci U S A. 2015;112(18):E2366-75.

28. Lee $H$, Han S, Kwon CS, Lee D. Biogenesis and regulation of the let-7 miRNAs and their functional implications. Protein Cell. 2016;7(2):100-13.

29. Martinez NJ, Ow MC, Reece-Hoyes JS, Barrasa MI, Ambros VR, Walhout AJ. Genome-scale spatiotemporal analysis of Caenorhabditis elegans microRNA promoter activity. Genome Res. 2008;18(12):2005-15.

30. de Lencastre A, Pincus Z, Zhou K, Kato M, Lee SS, Slack FJ. MicroRNAs both promote and antagonize longevity in C. elegans. Curr Biol. 2010;20(24): 2159-68.

31. Pincus Z, Smith-Vikos T, Slack FJ. MicroRNA predictors of longevity in Caenorhabditis elegans. PLoS Genet. 2011;7(9):e1002306.

32. Banerjee D, Chen X, Lin SY, Slack FJ. Kin-19/casein kinase lalpha has dual functions in regulating asymmetric division and terminal differentiation in C. elegans epidermal stem cells. Cell Cycle. 2010;9(23):4748-65.

33. Nakamura K, Kim S, Ishidate T, Bei Y, Pang K, Shirayama M, Trzepacz C, Brownell DR, Mello CC. Wnt signaling drives WRM-1/beta-catenin asymmetries in early C. elegans embryos. Genes Dev. 2005;19(15):1749-54.

34. Baldwin AT, Phillips BT. The tumor suppressor APC differentially regulates multiple beta-catenins through the function of axin and CKlalpha during $C$. elegans asymmetric stem cell divisions. J Cell Sci. 2014;127(Pt 12):2771-81.

35. Cadigan KM, Waterman ML: TCF/LEFs and Wnt signaling in the nucleus. Cold Spring Harb Perspect Biol 2012, 4(11).

36. Bhambhani C, Ravindranath AJ, Mentink RA, Chang MV, Betist MC, Yang YX, Koushika SP, Korswagen HC, Cadigan KM. Distinct DNA binding sites contribute to the TCF transcriptional switch in C. elegans and Drosophila. PLoS Genet. 2014;10(2):e1004133.

37. Arasu P, Wightman B, Ruvkun G. Temporal regulation of lin-14 by the antagonistic action of two other heterochronic genes, lin-4 and lin-28. Genes Dev. 1991;5(10):1825-33.

38. Feinbaum R, Ambros V. The timing of lin-4 RNA accumulation controls the timing of postembryonic developmental events in Caenorhabditis elegans. Dev Biol. 1999;210(1):87-95

39. Abrahante JE, Daul AL, Li M, Volk ML, Tennessen JM, Miller EA, Rougvie AE. The Caenorhabditis elegans hunchback-like gene lin-57/hbl-1 controls developmental time and is regulated by microRNAs. Dev Cell. 2003;4(5): 625-37.

40. Lin SY, Johnson SM, Abraham M, Vella MC, Pasquinelli A, Gamberi C, Gottlieb E, Slack FJ. The C elegans hunchback homolog, hbl-1, controls temporal patterning and is a probable microRNA target. Dev Cell. 2003;4(5):639-50.

41. Li M, Jones-Rhoades MW, Lau NC, Bartel DP, Rougvie AE. Regulatory mutations of mir-48, a C. elegans let-7 family MicroRNA, cause developmental timing defects. Dev Cell. 2005;9(3):415-22.

42. Lim LP, Lau NC, Weinstein EG, Abdelhakim A, Yekta S, Rhoades MW, Burge CB, Bartel DP. The microRNAs of Caenorhabditis elegans. Genes Dev. 2003; 17(8):991-1008.

43. Brenner S. The genetics of Caenorhabditis elegans. Genetics. 1974;77:71-94.

44. Watts JL, Phillips E, Griffing KR, Browse J. Deficiencies in C20 polyunsaturated fatty acids cause behavioral and developmental defects in Caenorhabditis elegans fat-3 mutants. Genetics. 2003;163(2):581-9.

45. Sharanya D, Thillainathan B, Marri S, Bojanala N, Taylor J, Flibotte S, Moerman DG, Waterston RH, Gupta BP: Genetic control of vulval development in Caenorhabditis briggsae. G3 (Bethesda) 2012, 2(12):1625-1641.

46. Grad Y, Aach J, Hayes GD, Reinhart BJ, Church GM, Ruvkun G, Kim J. Computational and experimental identification of $C$. elegans microRNAs. Mol Cell. 2003;11(5):1253-63.

47. Hofacker IL. Vienna RNA secondary structure server. Nucleic Acids Res. 2003; 31(13):3429-31.

\section{Publisher's Note}

Springer Nature remains neutral with regard to jurisdictional claims in published maps and institutional affiliations.
Ready to submit your research? Choose BMC and benefit from:

- fast, convenient online submission

- thorough peer review by experienced researchers in your field

- rapid publication on acceptance

- support for research data, including large and complex data types

- gold Open Access which fosters wider collaboration and increased citations

- maximum visibility for your research: over $100 \mathrm{M}$ website views per year

At BMC, research is always in progress.

Learn more biomedcentral.com/submissions 